\title{
Can Enterprise Architectures Reduce Failure in Development Projects?
}

\author{
Marijn Janssen \\ Delft University of Technology \\ M.F.W.H.A.Janssen@tudelft.nl \\ Bram Klievink \\ Delft University of Technology \\ A.J.Klievink@tudelft.nl
}

\begin{abstract}
Purpose: Scant attention has been given to the role of enterprise architecture in relationship to risk management in information system development projects. Even less attention has been given to the inter-organizational setting. The aim of this paper is to better understand this relationship.
\end{abstract}

Methodology: The relationship between EA and project failure/success is investigated by - through a workshop creating a retrospective view on the use of architectures in large and complex ICT-projects.

Findings: Failure factors can be grouped in organization network, people, process, product and technology categories. The findings show that a disappointingly limited number of public sector development projects make sufficient use of architecture as a risk management instrument. Architectures should both be considered as a riskmitigating instrument and as an organizational shaping routine to reduce project failure and manage risk in organization networks.

Research limitations: A single workshop with a limited number of participants was conducted. The findings need further refinement and generalization based on more empirical research investigating the relationship between architecture and project failure.

Implications: Architecture should give explicit consideration to risk management and help to draw attention to this. Governance mechanisms need be defined to ensure that the organizations and its members become aware of both architecture and risk management. Risk management and EA have similarities, as they are both an instrument and an organizational shaping routine.

Originality/value: Governments collaborate more and more in organizational networks and for that reason often multiple organizations are involved in information system project developments. Enterprise architecture as a risk mitigation instrument has not been given attention yet.

\section{Keywords}

Project failure, critical failure factors, risk management, enterprise architecture, information architecture.

Type: research paper

\section{INTRODUCTION}

Information system development is increasingly complex as systems go beyond the border of a single organization. Whereas in the past systems were developed and used within the boundaries of a single organization, nowadays organizations collaborate more and more in organizational networks (Milward and Provan, 1995). Public sector information systems are now developed for a user-base consisting of a large number of diverse organizations. Such networks are characterized by high degrees of interdependence among the organizations involved (Bruijn et al., 2010). When cooperating in networks, public agencies must not only manage their own activities, but they must also arrange and manage the relationships with other organizations and 
deal with requirements that are diverse and might - to some extent - even be conflicting. The inter-organizational nature results in a huge complexity, which easily results in exceeding the lead times, not delivering what was intended or might even result in complete failure. Even though a project might be successful from the point of view of one of the stakeholders, it may be viewed as a failure from the point of view of others.

There is a vast amount of studies on project failure (Lu et al., 2010, Yeo, 2002, Pinto and Mantel, 1990, Daniels and LaMarsh, 2007 ). Most of this literature is focused on identifying failure factors and looking for ways to improve success (Wallace et al., 2004). Despite the vast amount of literature, many information system development projects do not live up to the expectations (McAfee and Andrew, 2003). A paper in a Dutch ICT magazine provides an overview of 22 large project failures in both the public and the private sector. Each of these failures is responsible for wasting millions of Euro (Spoelstra, 2009). The need for reducing project failure rates has given rise to questions on whether enterprise architecture can be a suitable instrument to reduce project failure.

Flak et al. (2009) plea for more structured approaches with a focus on public values. Information architectures, enterprise architecture or architecture for short is a systematic and structured instrument to provide direction to the development of the ICT landscape and provide a holistic view at the organization (Janssen et al., 2009, Zachman, 1987, Armour et al., 1999). Enterprise architecture can be characterized as an emergent evolving endeavor shaped by institutional forces based on rational techniques (Hjort-Madsen, 2007). In an inter-organizational setting, architecture should help organizations to collaborate by providing overviews, standard and guidelines directing shared initiatives. Over the last decade, National Enterprise Architectures (NEA) and Enterprise architectures (EA) have been developed to guide organizations (Janssen and Hjort-Madsen, 2007, Peristera and Tarabanis, 2000). In the Netherlands, a variety of architectures exist. None of these have the explicit aim to reduce project failure or to mitigate development risks, although the architectures might include instruments to mitigate risks.

This research is aimed at understanding the existing role of architecture in relationship to project failure. The starting point of this research is the basic premise that project failure can be decreased by including risk management in enterprise architectures. The relationship between EA and project failure is investigated by creating a retrospective view on the use of EA in large and complex ICT-projects in the setting of collaborating organizations. A questionnaire is developed aimed at measuring the use of EA and risk management in these system development projects. During an interactive workshop, participants from various public organizations in the Netherlands were asked to fill in this survey for the projects they have experience with and to discuss the results.

This paper is structured as follows. In the next section, the background of project failure, risk management and architecture is described. This background is used to develop a measurement instrument, which in turn is used in the workshop to position projects in relation to EA and risk management approaches. This approach is discussed in the subsequent part. Finally, the research findings are discussed and conclusions draw.

\section{BACKGROUND}

\subsection{Failure factors}

Projects can be deemed failures from various views, including an inability to meet requirements, or when exceeding time and/or budget. Project failure can be viewed on a scale ranging from not delivering parts to complete failure in which almost all efforts and money is wasted. In this paper we investigate information system projects that have failed in the sense that the delivered products (if any) are not used and the system is abandoned.

Project failure has been extensively studies in ICT projects (Lu et al., 2010, Yeo, 2002, Pinto and Mantel, 1990, Daniels and LaMarsh, 2007 ). There are several categories of project failure, including people, process, product and technology (McConnell, 1996). Nelson (2007) uses this categorization to list classical project management mistakes. Table 1 extends the work of McConnell (1996) and Nelson (20070 for the public sector. A new category of organizational network failure factors is introduced, based on factors mentioned in public-sector projects (Lam, 2005, Janssen and Cresswell, 2005, McIvor et al., 2002, Dale and Goldfinch, 2006, Ebrahim and Irani, 2005 ). This new category accounts for the inter-organizational nature in which public organizations collaborate. Much of the factors originate from the variety in objectives and path-dependent situations of the 
organizations collaborating in networks. Their objectives, cultures and systems are likely to be different in one way or another. Furthermore, collaborating in networks requires clear responsibilities and allocation of roles (Janssen et al., 2006). An important part of managing collaborations are agreements, contracts, procedures and other instruments used to specify the relationship between the partners (e.g. Pongsiri, 2003). Not only do the roles in the project itself need to be specified, but also the roles and responsibilities in the inter-organizational setting. Agreements need to be negotiated as the costs and benefits are often spread over the various organizations.

Table 1. Overview of failure factors for inter-organizational development projects

\begin{tabular}{|c|c|}
\hline Category & Failure factors \\
\hline $\begin{array}{l}\text { Organizational } \\
\text { network }\end{array}$ & $\begin{array}{l}\text { Opposing aims and requirements } \\
\text { Organizational politics } \\
\text { Low levels of participation } \\
\text { Difference in technology readiness } \\
\text { Difference in (organizational) culture } \\
\text { Unclear overall program responsibilities } \\
\text { Absence of champion } \\
\text { Shifting goals }\end{array}$ \\
\hline People & $\begin{array}{l}\text { Undermined motivation } \\
\text { Individual capabilities } \\
\text { Working relationships } \\
\text { Absence of collaboration mechanism } \\
\text { Failure to deal with problems with employees } \\
\text { Adding people to a late project } \\
\text { No sponsorship } \\
\text { Change in stakeholders (contractors) }\end{array}$ \\
\hline Process & $\begin{array}{l}\text { Wasted time in the fuzzy front-end } \\
\text { Underestimate time, too limited scope, optimistic schedules } \\
\text { Insufficient risk management } \\
\text { Discussions about process dominate over project content } \\
\text { Scope creep } \\
\text { Contracting out } \\
\text { No business change } \\
\text { No user involvement }\end{array}$ \\
\hline Product & $\begin{array}{l}\text { Unclear shared objectives } \\
\text { Too ambitious requirements (e.g. performance, reliability) } \\
\text { Requirements gold-plating } \\
\text { Feature creep } \\
\text { Developers gold-plating } \\
\text { Research-oriented development }\end{array}$ \\
\hline Technology & $\begin{array}{l}\text { Silver-bullet } \\
\text { No proven technology available } \\
\text { Too many innovations } \\
\text { Overestimating savings } \\
\text { New practices entail new risks which are only discovered by use in practice } \\
\text { Switching tools during a project }\end{array}$ \\
\hline
\end{tabular}

\subsection{Risk management}

The large number of project failures and failure factors have resulted in risk management approaches to deal with the risks (Lyytinen et al., 1998, Nelson, 2007). Risk management deals with the identification, assessment, and prioritization and mitigation of risks to reduce the negative outcomes. Understanding software project failure and the risks involved can help in reducing the incidence of failure (Wallace et al., 2004). Risks are founded in possible incidents of failures that might occur with a certain likelihood. In general, risk management helps to identify, analyze and tackle software risks, implementation risks, project portfolio risks, or requirements risks (Lyytinen et al., 1998). 
There is a diversity of risk management methods which address similar problems with different methods (Lyytinen et al., 1998). Risk management methods make important recommendations like the use of project management, standards, involving users early on, and so on (Lyytinen et al., 1998) and the identification of success factors (Nelson, 2005, Nelson, 2007). There are many rational approaches based on decision theory which attempt to control the situation in order to mitigate risks. These often look at undesired outcomes, their variation in distribution, their likelihoods and impacts, and how to deal with them. This assumes that risk can be estimated in advance and that measures can be taken to avoid losses. This instrumental approach is useful and necessary, however, such an approach ignores the more socio-political aspects. Socio-political aspects like infrastructural, environment, human and social capital are important parts of e-government projects (Grimsley et al., 2007). A contrasting approach is to view risk management as an organizational attention shaping routine (Lyytinen et al., 1998). This approach is based on the idea that "risk management approaches fundamentally embody organizational routines of attention shaping which represent causal dependencies between managerial action and observed events" (Lyytinen et al., 1998 p. 234). Taking this approach should help decision-makers to get a better grip on situations and thereby help to avoid risks. The instrumental approach is similar to the view on architecture as a product, in which lists of failures are identified as part of the architecture and measures are suggested to mitigate them. In a similar vein, the organizational attention approach is similar to architecture governance, in which architecture is embedded in the organization.

\subsection{Enterprise architecture}

EA aims to bridge the gap between organizational and technology aspects (Zachman, 1987). It can describe or prescribe the dependencies among a large number of heterogeneous elements. In this way, it is an instrument to guide design decisions and provide direction for progressing towards the next generation of infrastructure (Janssen et al., 2009). EA should address the dependencies among a large number of heterogeneous elements at both the organizational and technical levels (Doucet et al., 2008). Traditionally, the purpose is to effectively align the strategies of enterprises with their business processes and the coordination of their resources (Zachman, 1987). EAs define and interrelate data, hardware, software, and communication resources, as well as the supporting organization required to maintain the overall physical structure required by the architecture (Richardson et al., 1990).

There can be found a wide variety of architectures in governments. IT architects and managers used numerous proverbs in conjunction with the term 'architecture'. A first distinction is the use of start architecture and enterprise architecture. Whereas Enterprise Architecture scopes the organization (enterprise) as a whole, whereas a start architecture refers to the initial architecture developed for a certain project of system. A start architecture provides guidance for project-level decision-making. Enterprise and start architectures are closely related. Furthermore, Iyer and Gottlieb (2004) make a distinction between two types of architectures.

1. Architecture-in-design: architecture is the outcome of a process of defining and modeling the architecture;

2. Architecture-in-operation: highlights the dependencies between organization and system components and provides the center of the architecture-in-design.

With others, Iyer and Gottlieb argue that an EA actually emerges as a result of implementing individual projects, which are guided by a start architecture. This brings us to the next distinction in architecture; the role of architecture as an instrument (Architecture_Working_Group, 2000) and architecture as a process giving directions to the development of a system (Spewak, 1992). Architecture as an instrument is a blueprint describing the relationships among the components in the system, whereas the latter view looks at how the architecture is used as an organizational routine to shape information projects. This distinction is similar to the distinction found in risk management methods; on the one hand in risk management there is an instrumental approach in which is looked at the (undesired) outcomes, which is similar to the architecture as a blueprint approach. On the other hand, there is the organizational shaping approach, which is similar to the development process approach. Architecture influences the design decisions and the investment behavior of an organization and in turn is influenced by behavior and design decisions. When viewed from this perspective the governance of the decision-making processes and organizational processes becomes a key concern of architecture. 


\section{RESEARCH APPROACH}

The goal of this exploratory research is to investigate the relationship between enterprise architecture and project risk management. This was done by organizing a session with government representatives involved in EA and ICT projects. In total, 15 session participants scored 14 different projects on the dimensions EA and risk in a matrix. Most of the projects concern inter-organizational system development projects in which organizations developed a mutual system to improve working in networks. The projects were aimed at accomplishing objectives like integrated service delivery, improved information exchange and business process management. Typically, many public organizations were involved and the system development was primarily executed by multiple private parties.

The session was facilitated by one of the authors and organized by a government representative. The session was held in October 2009 and the session participants had experiences with the ICT projects they were scoring. The participants included (potential) users, enterprise and information architects, and decision-makers. During the session, the main steps followed were:

1. Introduction and background;

2. Selection of projects: The projects were limited to those large and complex projects of which the participants had explicit knowledge due to their involvement in the projects. Furthermore both projects that have failed (the projects are on the list of failed project) and projects that were relatively successful (according to the participants) should be included;

3. Scoring the projects. All participants were asked to score the projects they had knowledge of, based on the questionnaire. There were 10 questions for EA and the same number of questions about risk management. For each questions one point could be gained, resulting in a maximum score of 10 for risk management and 10 for EA;

4. Discussion of scores. Each of the participants positioned the project they scored on a whiteboard displaying a matrix. All scores were discussed and in case of a deviation among scores for one project, consensus was sought;

5. Discussing relationship EA and risk management. The scores in the matrix were discussed by looking at each single axis first and then discussing the relationship between EA and risk management. Explicit attention was paid to explaining the results;

6. Conclusions and policy recommendations. Finally, the findings were summarized and participants were asked to support the conclusions. Based on the findings, a number of policy recommendations were drawn.

During the session, the emphasis was on mutual learning and understanding each other to facilitate open discussions.

\section{FINDINGS}

The projects were positioned in a matrix that is based on the use of EA and risk management. Participants were asked to fill in 20 survey questions based on their own experiences and involvement in the project. The survey questions were purposefully limited to the main aspects of EA and risk management; detailed questions were avoided as our aim was not to create an in-depth and extensive overview of EA and risk management, but to gain an indication about the actual use in past projects. We thereby avoided a situation in which participants would be guessing whether something would be the case or that the positioning and discussion would focus on details. The position of the projects should therefore be seen as an indication and not as an exact measure. The participants scored the projects in the EA/risk management matrix, resulting in the scored matrix, shown in figure 1.In the figure, grey circles refer to the projects that failed and are on the list of the Accountability Office in their analyses of failure of large and complex projects (Algemene_Rekenkamer, 2007, Algemene_Rekenkamer, 2008). 


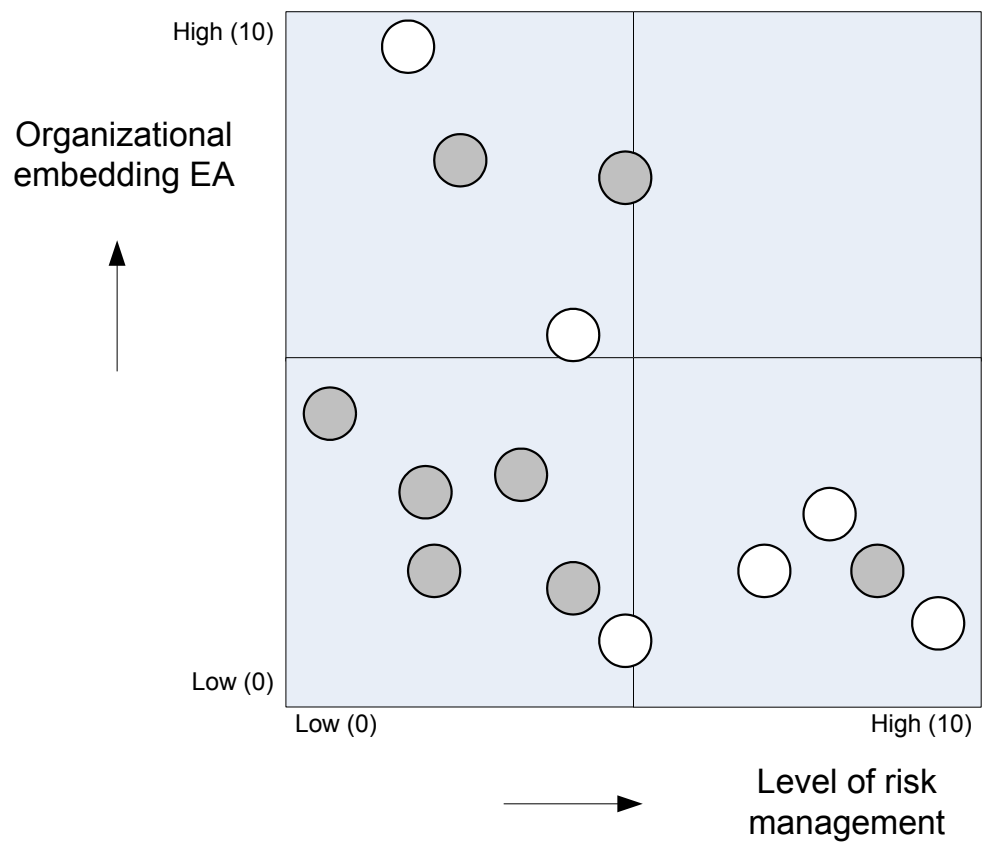

Figure 1: Scoring by the participants on the EA/risk matrix

In our approach, it was acceptable that several people would initially position the same project differently. The projects' positions were discussed with the participants and agreement was sought and found on the position of the projects. The deviation between these scores was limited and it was easy to find consensus about the final position of the projects in the matrix. The interpretation of the findings in the matrix was discussed and the positioning and findings did not surprise the participants.

The matrix shows that a disappointingly number of projects did not use either EA or risk management in a sufficient way. This could be attributed to the type of projects ranked in the workshop. A focus on successful projects might show another picture. Six out of 14 projects (43\%) are positioned in the lower left quadrant and no projects are found in the upper right quadrant, which would indicate it having high levels of risk management and EA. All of the projects in the lower left quadrant had an inter-organizational nature. Commonly mentioned factors were the lack of cross-organizational orchestration and the working in siloes. One participant commented that "at the end, the information could not be processed due to differences in software .... this was founded in the lack of communication among the organizations". Furthermore, it was suggested that the lack of orchestration and governance resulted in decision making at the operational level in the organization without looking whether the overall architecture expressed in standards, principles and models.

The participants were asked whether EA and risk management would be conflicting, i.e. that only one of the two could be used. The participants rejected this proposition, as most of them agreed that it would be possible to combine them. Furthermore, an example was given in which both aspects were given consideration at the same time. This example was used to prove that EA and risk management can co-exist. Some of the participants argued that EA will be strengthened by including risk management. The use of risk management will reinforce the other aspects of EA and the other way around.

The matrix shows that when a project has low scores on both EA en and risk management, the project is prone to failure. All projects that are entirely in the lower left quadrant failed. One person asked how it could be possible that so many of the projects were in this quadrant. In the discussion that followed, it was concluded that most projects started without any explicit check on the use of architecture and risk management techniques, as they were not mandatory. Often, project managers were found to be too optimistic concerning the feasibility and risk of the projects. 
The results illustrate that the use of EA in a project does not automatically guarantee success. Although there are examples of successful projects that are developed and implemented based on a sound architecture, there are also examples of projects that failed, despite the use of architecture. One possible explanation is that due to the use of architecture, more attention is given to the project which decreases the chance of failure.

Specifically when it comes to inter-organizational projects, a lack of organizational procedures to coordinate efforts among organizations can also easily result in project failure. The use of EA within the organizations in a network of partners needs to focus on the issues mentioned in table 1. Yet, most of these organizations have a lack of prior experience in mitigating risks in collaborations. Without a strong governance model, the individual organizations' past efforts on embedding EA within the organization can inhibit interoperability with other organizations that have pursued EA with the similar focus on the internal organization. Given the diversity of interests, goals, history and culture of organizations collaborating, EA architecture needs to be more stringent and may need to be made mandatory for the network of organizations to mitigate project risk. Some participants argued that architecture and governance should be intertwined, as architecture needs to be guided by good governance.

The use of risk management approaches and techniques helps to avoid failure, but again this alone is not sufficient to guarantee success. One participant indicated that 'some factors like resistance of certain parties and the use of unproven technology cannot be mitigated. The project will fail, despite having good risk management'. The participants indicated that an important factor to avoid failure is that the starting points should be good. The inability of solving failure factors at the beginning of a project will likely result in failure at the end.

During the discussion, the participants argued on whether EA did or did not provide any risk management methods and tools. The participants indicated that most EA do provide some methods and tools, therefore the score was never zero. Nevertheless, the support from EA for risk management was deemed poor as most EA did not provide explicit attention to this or it was limited to several lines or a small (sub)section in the architecture documentation, which is easily overlooked or neglected. One architect indicated that in his opinion, risk management should not be part of any EA. Instead, it should be part of project management methodologies. This resulted in much criticism of the others, as in their opinion EA should also be exploited as a type of risk mitigation instrument. As one participant said, "an architecture not considering the risks and potential failure of its use is a nightmare for everybody using the architecture".

\section{DISCUSSION AND IMPLICATIONS}

Project success and failure is elusive as projects are by their very nature risky. Many reasons are cited for their failure which can be categorized by people, process, product and technology (McConnell, 1996). We introduced a new category of inter-organizational failure factors which accounts for the inter-organizational complexities and nature in which public organizations collaborate. In inter-organizational situations there are stakeholders that are pluralistic and while presumably for supporting the project outcomes, nevertheless have their own objectives and requirements and ideas about how the projects should be implemented and executed. Furthermore, interorganizational settings might even more subject to politics, cultural variety and differences in technology knowledge and readiness. The broad range and variety of possible failure factors makes it difficult to mitigate the risk.

Risk management and enterprise architecture are intertwined in the execution of ICT-projects. Almost all session participants agreed that the enterprise architecture should give attention to risk management. Risk management should not be considered a separate discipline, but an integral part of the architecture and governance of the architecture. This finding is supported by Liu and Hwang (2003), who consider insufficient IT governance by the central government a failure factor. EA can be viewed as one type of risk management instrument that is complimentary to risk mitigation in project management approaches. One way to realize this is by including risk management as a separate view within an architecture, similar to how security and IT-service management is treated in architectures, including the Dutch national reference architecture. Also, a separate document, website or even a governmental program could be created to guide architects and project managers. This should preferably be a part of the national enterprise architecture, as this architecture serves as the basis for other architectures and 
this would ensure the necessary visibility. Furthermore, risk managers should be involved in the national EA efforts to ensure that essential risk management elements are included in EA.

In future large and complex projects, much more attention should be given to risk management. General risk management approaches should be used and risks should be identified and mitigated. The table presented at the beginning of this paper contains commonly found failure factors can be used for identifying possible risks. In addition, there should be regular reviews in which the risks are assessed and compliance with the architecture is checked. The participants agreed that risk management should not result in thick documents, but in practical and ready to use recommendations. The government should have risk managers that regularly assess large and complex projects to help to avoid failure. This could be done by using Gateway Review Process (GRP) which are carried out at key decision points by panels of experts, independent of the project. GRPs are not part of the project organizations and reside under the governance board. The reviews provide input for both the governance board as well as the project management.

The separation of responsibilities in project management was found to be important having implications on the allocation of responsibilities in future projects. Instead of project managers, another person should be responsible for risk management in large and complex projects. This is preferably a person, who is not too heavily involved in the daily operations, but does have knowledge and understanding of the project and the authority that is accepted by the project manager. This could be a risk manager, but could also be an architect who regularly assesses the risks and looks for mitigation mechanisms. These risk managers could be part of a separate government program about risk management in order to avoid the mixing up of project and risk management responsibilities. They should be separated as they serve different purposes. The aims of project management, such as staying on track, avoid spending of budget might be conflicting with the aim of minimizing risks, which measure might delay progress and might need the spending of money.

Both the use of EA and risk management techniques and tools were voluntary in the projects investigated in this study. Project managers could too easily deviate from EA principles and best practices, and could manage a project without having assessed risks and implemented any risk controls. This kind of behavior was viewed as undesirable and the participants inclined towards favoring the mandatory use of EA and risk management. This does not mean that project managers should follow all prescriptions and will be confronted with more bureaucracy and red tape, instead they should be given the freedom to motivate a deviation and ask for approval by a governance board.

Broader issues of technical, political, and organisational origin are of equal importance in e-government (Sarikas and Weerakkody, 2007). Despite having a good architecture and risk management, a project might fail when the conditions for success are not met. Conditions can be found on a wide variety of areas, like budget, commitment of leaders, resistance and so on (see for example Nelson, 2007). These types of conditions should be met in advance, as risk mitigation during the project might not work. An integrated approach to dealing with the failure factors of all categories should be employed, as these are often interdependent.

In addition to including risk management in EA, governance mechanisms need to be defined as well. This should include organizational routines and procedures to mitigate risks and to use the EA at the start of a project. Furthermore, all project member and participants should be aware of possible failure. This refers to the need of organizational attention shaping routine as mentioned by (Lyytinen et al., 1998). In this way risk management should become an integral part of day-to-day operations and behavior of organizational members.

\section{CONCLUSIONS}

There are a large number of ICT-project failure factors which can be grouped in the following categories; organization network, people, process, product and technology and complicates inter-organizational development projects. This explorative research started with the basic premise is that project failure can be decreased by including risk management in enterprise architectures. The relationship between enterprise architecture and project management was investigated by creating a retrospective view on the use of architecture and risk management in 14 projects. The results confirmed the initial expectation that architecture is hardly used as a risk mitigation instrument. Furthermore, many of the large and complex projects did not give any attention at all to EA 
or to risk management. The findings suggest that projects giving less attention to EA or risk management have a higher failure rate.

This results in the recommendation to include risk management in EAs. Especially, the national reference architecture should give explicit consideration to risk management and help to draw the attention to this. In addition, governance mechanisms should be defined to ensure that the all organizations and its members become aware of both architecture and risk management. Risk management and EA are similar in the sense that both should be embedded in the organization. EA is related to risk management as it is often used as a change management instrument to guide projects. The straightforward lesson is that there is a need risk management and EA instruments that are supported by organizational shaping routines.

In organizational networks, typical failure factors are related to the variety of objectives, cultures and systems which are likely to differ between organizations. Collaborating in networks requires clear responsibilities and allocation of roles and the stringent use of architecture. Mandatory use of EA building blocks, principles and standards will likely improve the design of systems that are interoperable and can be used to support crossorganizational processes. Architecture can be used to govern the inter-organizational situation and should go beyond the own organizational boundaries. Information system development by collaborating organizations might have higher failure rates than development within organizations. Therefore, their peculiar characteristics and failures rates need to be further researched.

\section{FUTURE RESEARCH}

The reduction of failure and the use of enterprise architecture is a source for many avenues or research. The particular complexities of inter-organizational setting needs further research attention. Which are the main risks and who can they be dealt with. EA and risk management are related as both can be implemented as instruments and organizational shaping routines. Both can potentially profit from each other and are complimentary. Yet, the exact relationship is unclear and needs further research attention.

Drawing on the limitations of this study we have several further research suggestions. First, only one session was held, and although the participants and projects were selected with care, they may not adequately represent the whole population of participants and projects. We suggest the generalization of the findings using a large random sample. We did, however, feel that our findings were corroborated by the fact that many of the failed projects found in our study were also identified as such by the National Accountability Office. Also cross-country and cross-sector studies can advance our insight.

Second, we primarily used the experiences of the session participants and did not look at EAs themselves. The EA used in a project might have included risk mitigation approaches and practices. The cause of not using the EA might be on the governance level, due to a lack of procedures and routines to use EA. As such we recommend the detailed analysis of the relationship between EA, governance and project failure. Various governance mechanisms should be investigated and their effects evaluated.

Finally, we used a limited number of questions to position the projects. This was due to time limitation within the session, and matched our aim to have only a rough indication instead of focusing on all kinds of details. We recommend the development of more detailed and comprehensive questionnaires and checklists to evaluate. These can be developed based on the questionnaire presented in this paper. This comprehensive list could be used to assess whether there is a relationship between certain factors and project failure. The results of such a comprehensive analysis could be used to let decision makers, and risk and project managers explicitly focus on the most important factors when attempting to reduce project failures.

\section{REFERENCES}

ALGEMENE_REKENKAMER 2007. Lessen uit ICT-projecten bij de overheid - Deel A. 's-Gravenhage: National Accountability office.

ALGEMENE_REKENKAMER 2008. Lessen uit ICT-projecten bij de overheid - Deel B. 's-Gravenhage: National Accountability office. 
ARCHITECTURE_WORKING_GROUP 2000. IEEE Std 1471-2000 Recommended Practice Architectural Description of Software-Intensive Systems. In: COMMITTEE, I. S. E. S. (ed.). Washington DC: IEEE Standard 1471-2000.

ARMOUR, F. J., KAISLER, S. H. \& LIU, S. Y. 1999. A big-picture look at Enterprise Architecture. IEEE IT Professional, 1, $35-42$.

BRUIJN, H. D., HEUVELHOF, E. T. \& VELD, R., IN 'T 2010. Why Project Management Fails in Complex Decision Making Processes, Heidelberg, Springer.

DALE, T. \& GOLDFINCH, S. 2006. Dangerous Enthusiasms: E-government, Computer Failure and Information Systems Development Otago University Press.

DANIELS, C. B. \& LAMARSH, W. J. 2007 Complexity as a Cause of Failure in Information Technology Project Management. IEEE International Conference onS ystem of Systems Engineering, 2007 (SoSE '07). IEEE.

DOUCET, G., GØTZE, J., SAHA, P. \& BERNARD, S. 2008. Coherency Management: Using Enterprise Architecture for Alignment, Agility, and Assurance. Journal of Enterprise Architecture, 4, 9-20.

EBRAHIM, Z. \& IRANI, Z. 2005 e-government adoption: architecture and barriers. Business Process Management Journal, $11,589-611$.

FLAK, L. S., DERTZ, W., JANSEN, A., KROGSTIE, J., SPJELKAVIK, I. \& ØLNES, S. 2009. What is the value of eGovernment - and how can we actually realize it? Transforming Government: People, Process and Policy, 3, 220226.

GRIMSLEY, M., MEEHAN, A. \& TAN, A. 2007. Evaluative design of e-government projects: A community development perspective. Transforming Government: People, Process and Policy, 1, 174-198.

HJORT-MADSEN, K. 2007. Institutional patterns of enterprise architecture adoption in government. Transforming Government: People, Process and Policy, 1, 333-349.

IYER, B. \& GOTTLIEB, R. M. 2004. The Four-Domain Architecture: An approach to support enterprise architecture. IBM Systems Journal, 43, 587-597.

JANSSEN, M., CHUN, S. A. \& GIL-GARCIA, J. R. 2009. Building the next generation of digital government infrastructures Government Information Quarterly, 26, 233-237.

JANSSEN, M. \& CRESSWELL, A. M. 2005. An enterprise application integration methodology for e-government. Journal of Enterprise Information Management, 18, 531-547.

JANSSEN, M., GORTMAKER, J. \& WAGENAAR, R. W. 2006. Web service orchestration in public administration: Challenges, roles and growth stages. Information Systems Management, 23, 44-55.

JANSSEN, M. \& HJORT-MADSEN, K. 2007. Analyzing Enterprise Architecture in National Governments: The Cases of Denmark and the Netherlands. In: SPRAGUE, R. (ed.) 40th Annual Hawaii International Conference on System Sciences (HICSS'07). Big Island, Hawaii: IEEE.

LAM, W. 2005. Barriers to e-government integration. The journal of Enteprise Information Management, 18, 511-530.

LIU, S. \& HWANG, J. D. 2003. Challenges to transforming IT in the US government. IT Professional, 5, 10-15.

LU, X., LIU, H. \& YE, W. 2010. Analysis failure factors for small \& medium software projects based on PLS method The 2nd IEEE International Conference on Information Management and Engineering (ICIME). IEEE.

LYYTINEN, K., MATHIASSEN, L. \& ROPPONEN, J. 1998. Attention Shaping and Software Risk- A Categorical Analysis of Four Classical Risk Management Approaches. Information Systems Research, 9, 233-255.

MCAFEE \& ANDREW 2003. When too much IT knowledge is a dangerous thing. MIT Sloan Management review, 83-89.

MCCONNELL, S. 1996. Rapid Development, Microsoft Press.

MCIVOR, R., MCHUGH, M. \& CADDEN, C. 2002. Internet technologies: supporting transparency in the public sector. International Journal of Public Sector Management, 15, 170-187.

MILWARD, H. B. \& PROVAN, K. G. 1995. A preliminary theory of network effectiveness: A comparative study of four community mental health systems. Administrative Science Quarterly, 40, 1-33.

NELSON, R. R. 2005. Project Retrospectives: Evaluating Project Success, Failure, and Everything in Between. MIS Quarterly Executive, 4, 361-372.

NELSON, R. R. 2007. IT project Management: Infamous failures, classic mistakes and best practices. MISQ Executive, 6, 67-78.

PERISTERA, V. \& TARABANIS, K. 2000. Towards an enterprise architecture for public administration using a top-down approach. European Journal of Information Systems, 9, 252-260.

PINTO, J. K. \& MANTEL, S. J., JR 1990. The causes of project failure. IEEE Transactions on Engineering Management, 37 $269-276$.

PONGSIRI, N. 2003. Public-Private Partnerships in Thailand: A Case Study of the Electric Utility Industry. Public Policy and Administration, 18, 69-90.

RICHARDSON, L., JACKSON, B. M. \& DICKSON, G. 1990. A Principle-Based Enterprise Architecture: Lessons From Texaco and Star Enterprise. MIS Quarterly, 14, 385-403. 
SARIKAS, O. D. \& WEERAKKODY, V. 2007. Realising integrated e-government services: a UK local government perspective. Transforming Government: People, Process and Policy, 1, 153-173.

SPEWAK, S. H. 1992. Enterprise architecture planning. Developing a blueprint for data, applications and Technology, New York, John Wiley.

SPOELSTRA, J. 2009. Spraakmakende ICT-fiasco's. Computable. VNU.

WALLACE, L., KEILB, M. \& RAI, A. 2004. Understanding software project risk: a cluster analysis. Information \& Management 42 (2004) 115-125, 42.

YEO, K. T. 2002. Critical failure factors in information systems projects. International Journal of Project management, 20, 241-246.

ZACHMAN, J. A. 1987. A Framework for Information Systems Architecture. IBM Systems Journal, 26, 276-292. 